\title{
Successful surgical treatment of an inflammatory lesion associated with new-onset refractory status epilepticus
}

\author{
Csaba Juhász, M.D., Ph.D., ${ }^{1,2,6,7}$ Amy Buth, M.S., ${ }^{3,7}$ Diane C. Chugani, Ph.D., ${ }^{1,4,6}$ \\ William J. Kupsky, M.D., ,,7 Harry T. Chugani, M.D., ${ }^{1,2,4,6}$ A ashit K. Shah, M.D., ${ }^{2}$ \\ AND SANDEEP MitTal, M.D., F.R.C.S.C. ${ }^{3,7}$
}

Departments of ${ }^{1}$ Pediatrics, ${ }^{2}$ Neurology, ${ }^{3}$ Neurosurgery, ${ }^{4}$ Radiology, and ${ }^{5}$ Pathology, Wayne State University; ${ }^{6}$ PET Center and Translational Imaging Laboratory, Children's Hospital of Michigan; and ${ }^{7}$ Karmanos Cancer Institute, Detroit, Michigan

\begin{abstract}
New-onset refractory status epilepticus (NORSE) has high morbidity and mortality. The authors describe the successful surgical treatment of a 56-year-old man presenting with NORSE. Magnetic resonance imaging showed a left temporal lobe lesion suspicious for a low-grade tumor, while PET imaging with the alpha[ $\left[{ }^{11} \mathrm{C}\right] \mathrm{methyl}-\mathrm{L}$-tryptophan (AMT) radiotracer showed increased cortical uptake extending beyond this lesion and partly overlapping with epileptogenic cortex mapped by chronic intracranial electroencephalographic monitoring. Resection of the epileptic focus resulted in long-term seizure freedom, and the nonresected portion of the PET-documented abnormality normalized. Histopathology showed reactive gliosis and inflammatory markers in the AMT-PET-positive cortex. Molecular imaging of neuroinflammation can be instrumental in the management of NORSE by guiding placement of intracranial electrodes or assessing the extent and severity of inflammation for antiinflammatory interventions. (http://thejns.org/doi/abs/10.3171/2013.3.FOCUS1336)
\end{abstract}

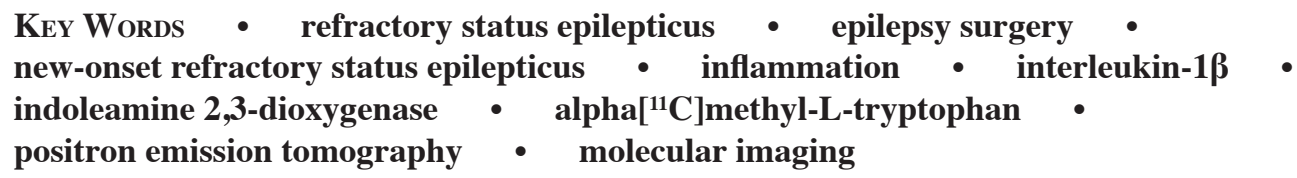

I $\mathrm{N}$ adults with new-onset focal seizure(s), cranial CT or MRI often reveals an epileptogenic lesion. In such cases, the lesion is often suspicious for an underlying neoplasm, although nonneoplastic conditions can also mimic brain tumors. In some adults, seizures occur as NORSE. ${ }^{11,17,26}$ This disorder is often characterized by CSF pleocytosis and lack of an obvious underlying cause. In some cases, seizures are preceded by a febrile illness. Magnetic resonance imaging may show signal abnormalities, often multifocal, on T2-weighted and FLAIR sequences. Treatment is difficult, and morbidity and mortality are high. The etiology of this condition is unclear; an underlying inflammatory process, such as viral encephalitis or an autoimmune condition, is frequently assumed but rarely can be proven. It is also often uncer-

\footnotetext{
Abbreviations used in this paper: AMT $=$ alpha $\left[{ }^{11} \mathrm{C}\right]$ methyl-Ltryptophan; $\mathrm{CD}=$ cluster of differentiation; $\mathrm{EEG}=$ electroencephalography; GFAP = glial fibrillary acidic protein; IDO = indoleamine 2,3 -dioxygenase; IL-1 $\beta=$ interleukin-1 $\beta$; IL-1R1 = receptor of IL-1 $\beta ;$ NORSE $=$ new-onset refractory status epilepticus.
}

tain whether the observed MRI abnormalities represent the causes or consequences of severe seizures. Here we present the clinical history, neuroimaging, electrophysiological, and histopathological findings, and long-term posttreatment follow-up of an adult treated for NORSE associated with an inflammatory brain lesion. We demonstrate how the use of AMT-PET imaging facilitated the diagnosis and assisted with successful treatment after detecting tissue with seizure-associated inflammation. AMT-PET is an imaging modality that can effectively detect epileptic foci and lesions such as tumors due to AMT accumulation resulting from increased tryptophan transport and metabolism via the inflammatory and immunosuppressive kynurenine pathway., ${ }^{4,514-16}$

\section{Case Report}

A 56-year-old right-handed man with a history of high blood pressure presented with sudden-onset progressive headache, followed by new-onset complex partial seizures 3 days later. There was no history of fever. On 
admission, general and neurological examinations were normal, except for fluctuating fluent dysphasia. Cranial MRI showed a nonenhancing lesion in the left temporal lobe, hyperintense on T2-weighted and FLAIR sequences, suspicious for a low-grade glioma (Fig. 1). One week later, he had a generalized seizure and, despite aggressive treatment, developed nonconvulsive partial status epilepticus nonresponsive to maximal doses of 4 antiepileptic drugs and intermittent intravenous benzodiazepines to treat breakthrough seizures. Initial CSF analysis showed $0 \mathrm{WBC} / \mathrm{mm}^{3}, 1 \mathrm{RBC} / \mathrm{mm}^{3}$, normal protein and glucose levels, and negative polymerase chain reaction for herpes simplex virus 1 and 2 . Upon arrival to our institution, continuous video-EEG monitoring showed periodic epileptiform discharges from the left temporal region with frequent electroclinical seizures resulting in episodic fluent aphasia. AMT-PET imaging was performed after obtaining informed consent and showed a relatively large cortical region of increased uptake within and adjacent (mostly posterior) to the MRI-defined lesion (Fig. 1).

Because of the persistent drug-resistant seizures (about 30 per day) and presence of focal MRI-defined abnormalities suspicious for an underlying glioma, the patient underwent a 2-stage epilepsy surgery with implantation of intracranial electrodes over the left frontotemporoparietal cortex 4 days after the PET scanning (Fig. 2A). A small image-guided biopsy of the MRI-defined lesion was performed prior to subdural grid implantation. Intracranial EEG monitoring showed frequent seizures emanating from the posterior aspect of the lateral temporal neocortex. Preliminary histological analysis from the tissue biopsy showed prominent astrocytosis thought to be related to an underlying or adjacent low-grade neoplasm.
After 3 days of extraoperative intracranial EEG monitoring and eloquent cortex mapping, the patient underwent volumetric resection of the lesion and surrounding epileptogenic zone in the temporal cortex (Fig. 1). The mesial temporal lobe structures were preserved as they were not involved in the seizures. Postoperatively, the patient recovered well, with residual receptive language deficits that improved over 1 year. Since having surgery 3 years ago, he has remained seizure free and has a mild residual receptive dysphasia. Follow-up MRI showed no recurrence of the lesion. Likewise, AMT-PET performed 3 months after surgery showed normalization of AMT uptake (Fig. 1) and remained unchanged at 18 months.

Immunological study showed absent anti-nuclear, anti-double-stranded DNA, anti-glutamic acid decarboxylase, anti-Hu, and anti-voltage-gated potassium channel antibodies. Likewise, a comprehensive paraneoplastic evaluation was negative.

Final histopathological evaluation of the biopsy specimen (obtained prior to subdural grid implantation) and the resected epileptic tissue showed recent neuronal necrosis, florid reactive astrocytosis (GFAP immunostaining, Fig. 2B), microglial activation (CD68 immunostaining), and sparse lymphocytic inflammation (CD45 immunostaining) without evidence of viral inclusion, cytopathic effect, or underlying neoplasm. Resected epileptic tissue was carefully divided and identified based on intracranial EEG and PET findings. The individual tissue blocks were studied for expression of IDO (the initial and rate-limiting enzyme of the kynurenine pathway), the inflammatory marker IL-1 $\beta$, and its receptor IL-1R1; these expressions were correlated with electrophysiological and neuroimaging findings. We noted strong coexpression
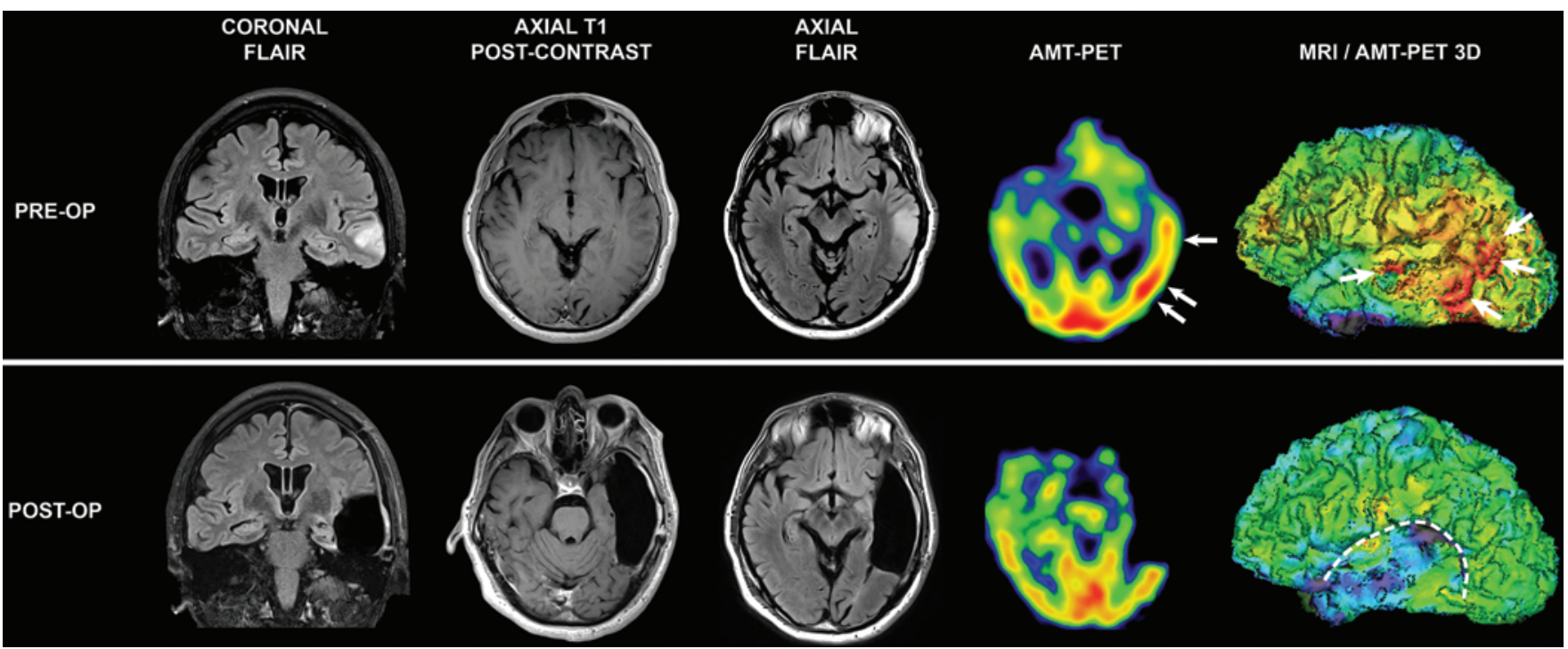

FIG. 1. Pre- and postoperative MRI and AMT-PET scans. Upper: Preoperative FLAIR and T1-weighted postcontrast images showing a nonenhancing hyperintense left midtemporal lesion. On PET images, increased AMT uptake is noted in the left mid- and posterior temporal cortex with partial overlap with the MRI-defined lesion. The regions with the highest AMT uptake in the mid- and posterior temporal areas are indicated with arrows (high AMT uptake in visual cortex is symmetrical, physiological). Lower: Follow-up MR images 3 months after prolonged intracranial EEG-guided resection of left lateral temporal lobe show the resection cavity with no residual lesion and preservation of mesial temporal lobe structures. Follow-up AMT-PET scan shows absence of AMT uptake in the resection cavity and normalization of AMT uptake in the residual posterior temporal region behind the resection cavity. 

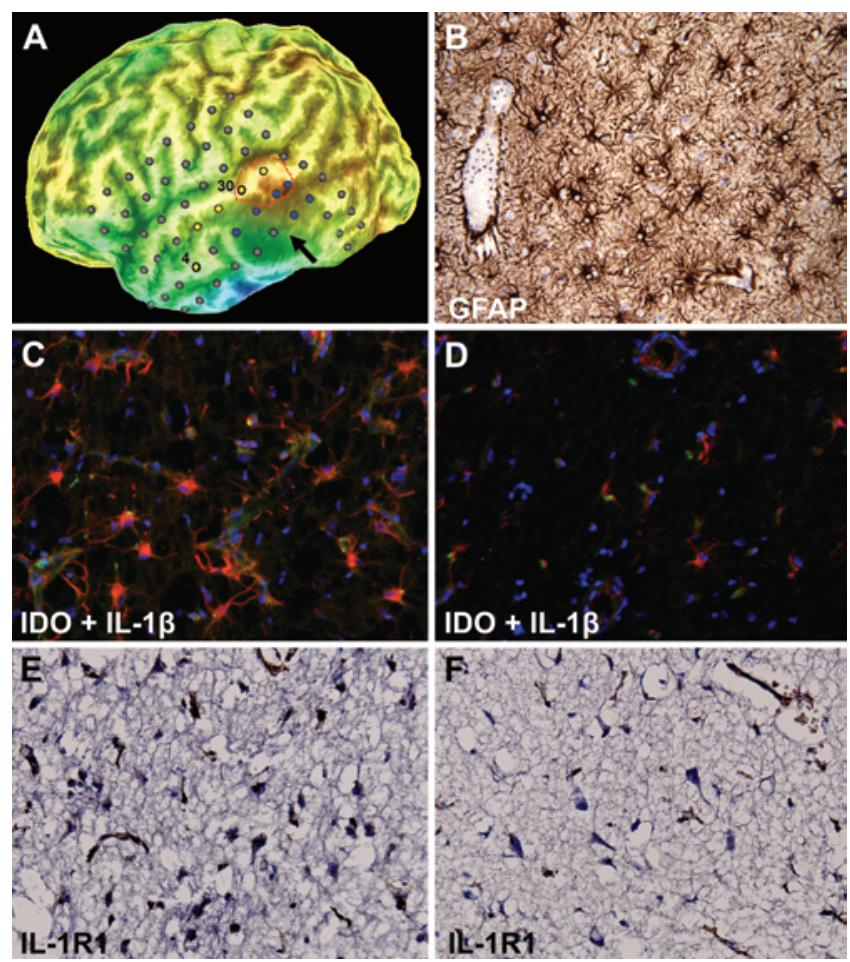

FIG. 2. Immunostaining findings of resected epileptogenic tissue. A: Curvilinear $3 \mathrm{D}$ reconstruction of the brain $(5 \mathrm{~mm}$ under the cortical surface to emphasize gyri and sulci) using the preoperative volumetric MRI with overlay of intracranial subdural electrodes. The arrow indicates the MRI-defined lesion in the subcortical area, whereas the outlined region in red corresponds to the highest AMT uptake in the superior temporal gyrus. Electrodes in yellow represent seizure onset, and those in blue showed rapid seizure propagation. Electrodes No. 30 (superior temporal gyrus) and No. 4 (middle temporal gyrus) are labeled because the cortex under these electrodes was sampled for immunohistochemistry (see below). B: GFAP immunohistochemistry showing extensive reactive astrocytes in the image-guided biopsy obtained from the nonenhancing, T2-weighted/FLAIR hyperintense lesion prior to implantation of intracranial electrodes. C: Strong IDO (red) and IL$1 \beta$ (green) coexpression in tissue obtained from electrode No. 30 corresponding to an AMT-positive region involved in seizure onset in the superior temporal gyrus. $\quad$ D: In contrast, there is sparse IDO and IL-1 $\beta$ coexpression in the tissue obtained from electrode No. 4 corresponding to an AMT-negative region involved in seizure onset in the anterolateral temporal cortex. Nuclei are stained in blue by DAPI counterstaining. $\quad E$ and $F$ : Immunostaining for IL-1R1 in the same regions again demonstrating higher expression in the AMT-positive tissue (electrode No. 30; E) compared with AMT-negative tissue (electrode No. 4; F). Original magnification $\times 20(B-F)$.

of IDO and IL-1 $\beta$ under temporal electrodes involved in seizure onset, showing an increased AMT uptake on PET (Fig. 2C), whereas IDO and IL-1 $\beta$ coexpression was sparse at the anterior temporal cortex (Fig. 2D); the latter was also involved in some seizures but did not have increased AMT uptake. Similarly, there was increased expression of IL-1R1 in the AMT-positive area (Fig. 2E and F).

\section{Discussion}

The clinical features described in this 56-year-old man fit the diagnosis of NORSE, although there were some unusual features. Notably, our patient had a single left temporal lesion resembling a low-grade neoplasm, while in most cases brain abnormalities on MRI and epileptiform activity on EEG tend to be multifocal. ${ }^{11,26}$ However, histopathology revealed inflammatory changes without any evidence of tumor. There have been several conditions described in the literature that share features with NORSE, including fever-induced refractory epileptic encephalopathy and others (reviewed by Ismail and Kossoff), ${ }^{11}$ but these affect younger populations (mostly children, rarely young adults) and a febrile illness is almost unanimously present before disease onset. In all of these conditions, abolition of status epilepticus is difficult, mortality is high, and neurocognitive outcome is often devastating. Surgical treatment is usually not considered due to the multifocal abnormalities. In contrast, our patient underwent emergency surgery and recovered with complete cessation of seizures and minimal neurological symptoms.

The AMT-PET findings played an important role in the diagnosis and management of our patient. AMT is a PET tracer, originally developed for mapping cerebral serotonin synthesis, which is not a substrate of the enzymes involved in protein synthesis. ${ }^{8,23}$ Subsequent studies in patients with partial epilepsy have suggested that AMT may accumulate in epileptic cortex and in epileptogenic lesions as a result of increased metabolism via the inflammatory kynurenine pathway. ${ }^{5}$ This pathway plays a limited role in the normal brain but can be significant under inflammatory conditions, mostly via upregulation of IDO. ${ }^{9}$ In the presented case, increased AMT accumulation extended considerably beyond the nonenhancing MRI-defined lesion, mostly into the posterior temporal cortex (Fig. 1). While most low-grade gliomas accumulate AMT, ${ }^{15}$ increased tracer uptake usually does not extend far beyond the lesion; ${ }^{16}$ thus, this PET finding made presence of a low-grade glioma less likely. Rather, increased AMT uptake around nonneoplastic lesions is highly suspicious for epileptic cortex, as it has been seen in perituberal cortex in children with tuberous sclerosis complex. ${ }^{1}$ The advantage of AMT over 2-deoxy-2 $\left[{ }^{8} \mathrm{~F}\right]$ fluoro-D-glucose as a PET radiotracer is its high specificity to detect epileptic cortex via focal radiotracer accumulation in the interictal state. ${ }^{14}$ Therefore, the relatively extensive temporal cortical AMT-PET abnormality, together with the electroclinical symptoms in our patient, prompted us to map the ictal onset zone with long-term subdural EEG monitoring before resection of a large portion of the left temporal lobe, which helped to maximize the chance of seizure freedom. This strategy was indeed successful, as the patient has remained seizure free over a 3-year follow-up period.

Histopathology of the resected epileptic tissue showed reactive gliosis and inflammation, which was present specifically in the AMT-accumulating tissue. High expression of IDO in the specimen suggested activation of the inflammatory kynurenine pathway and increased conversion of tryptophan to kynurenine metabolites as a result. ${ }^{5}$ Proinflammatory cytokines, such as IL-1 $\beta$ or tumor necrosis factor- $\alpha$, can potentiate induction of IDO..$^{10,21}$ IL$1 \beta$, along with other cytokines, plays an important role in 
the mechanisms of hyperexcitability involved in experimental seizure models. ${ }^{24}$ Cortical tubers resected to alleviate seizures showed signs of a chronic inflammatory response, including expression of a variety of markers such as IL-1 $\beta$ and its signaling receptor IL-1R1, components of the complement cascade, CD68-reactive macrophage infiltration, and expression of molecules (such as tumor necrosis factor- $\alpha$ ) involved in cytokine signaling. ${ }^{2,19}$ Epileptogenic focal cortical dysplasia Type II (but not Type I) also showed prominent expression of IL- $1 \beta$, components of the complement cascade, and perivascular and parenchymal CD3+ T lymphocytes (with a predominance of CD8+ cytotoxic/suppressor T cells), thus supporting involvement of different inflammatory pathways in these developmental lesions. ${ }^{12}$ This expression pattern appears to coincide with the pattern of increased AMT uptake seen in focal cortical dysplasia subtypes. ${ }^{6}$ Expression of IL-1 $\beta$ and IL-1R1 was also seen in specimens obtained from epileptogenic glioneuronal tumors, with widespread expression in multiple cell types including neurons, astrocytes, and microglia. ${ }^{22}$ Seizure-induced brain inflammation and IL-1 $\beta$ release are also associated with transient blood-brain barrier impairment. ${ }^{18}$ Therefore, increase of AMT uptake and trapping in epileptic tissue may be related to increased tryptophan transport (due to blood-brain barrier defect) and metabolism of tryptophan to L-kynurenine (due to IDO activity), respectively. Coexpression of IL-1 $\beta$, IL-1R1, and IDO in AMT-accumulating cortex in specimens obtained from our patient is consistent with the notion that increased AMT uptake shown by PET imaging of the epileptic brain may serve as a biomarker of immune activation. ${ }^{3}$ Comparison of the intracranial EEG and PET findings also suggested that the inflammatory changes extended beyond the epileptogenic region. Postsurgical reversal of increased AMT uptake in nonresected cortex in the posterior temporal region (which was not involved in seizure onset) suggests that some of the AMT-PET abnormalities were either seizure induced or represented reversible inflammation not inducing epileptogenesis.

The etiology of seizures in this patient remains unknown, as is the case with most patients with NORSE. However, there is an increasing body of evidence demonstrating that release of IL-1 $\beta$ and other proinflammatory cytokines can be both a cause and a consequence of severe seizures, thus playing a central role in inflammationmediated seizures and status epilepticus..$^{20,25}$ Since status epilepticus in NORSE is resistant to standard antiepileptic treatment and the incidence of mortality is especially high in adults, there is an urgent need for novel therapeutic approaches. Our case demonstrates that surgery is an option when neuroimaging and electrophysiological data indicate a relatively limited unilateral brain abnormality. In cases with multifocal abnormalities, pharmacological approaches might be the only option. The results with wide-spectrum immunomodulatory treatment regimens have been disappointing; therefore, exploration of new antiinflammatory strategies is warranted.,20 Molecular imaging with AMT, or other imaging approaches targeting molecular mechanisms associated with neuroinflammation, ${ }^{13}$ can offer a noninvasive way to assess presence, severity, and extent of seizure-associated inflammatory changes in the epileptic brain. These modalities could be instrumental not only when surgery is being considered but also in clinical trials as biomarkers when testing novel antiinflammatory approaches.

\section{Disclosure}

The authors report no conflict of interest concerning the materials or methods used in this study or the findings specified in this paper.

The study was supported by a grant (R01 CA123451 to C.J.) from the National Cancer Institute, Start-up Funds (Wayne State University School of Medicine to S.M.), and a Strategic Research Initiative Grant from the Barbara Ann Karmanos Cancer Institute (to S.M. and C.J.).

Author contributions to the study and manuscript preparation include the following. Conception and design: Mittal, Juhász, Shah. Acquisition of data: Mittal, Juhász, Buth, Kupsky, Shah. Analysis and interpretation of data: Mittal, Juhász, Buth, Kupsky, Shah. Drafting the article: Mittal, Juhász, Shah. Critically revising the article: Mittal, Juhász, D Chugani, Kupsky, H Chugani, Shah. Reviewed submitted version of manuscript: all authors. Approved the final version of the manuscript on behalf of all authors: Mittal. Administrative/technical/material support: Mittal, Juhász. Study supervision: Mittal, Juhász.

\section{References}

1. Asano E, Chugani DC, Muzik O, Shen C, Juhász C, Janisse $\mathrm{J}$, et al: Multimodality imaging for improved detection of epileptogenic foci in tuberous sclerosis complex. Neurology 54:1976-1984, 2000

2. Boer K, Jansen F, Nellist M, Redeker S, van den Ouweland AM, Spliet WG, et al: Inflammatory processes in cortical tubers and subependymal giant cell tumors of tuberous sclerosis complex. Epilepsy Res 78:7-21, 2008

3. Chugani DC: $\alpha$-methyl-L-tryptophan: mechanisms for tracer localization of epileptogenic brain regions. Biomarkers Med 5:567-575, 2011

4. Chugani DC, Chugani HT, Muzik O, Shah JR, Shah AK, Canady A, et al: Imaging epileptogenic tubers in children with tuberous sclerosis complex using alpha-[11C]methyl-L-tryptophan positron emission tomography. Ann Neurol 44:858866, 1998

5. Chugani DC, Muzik O: Alpha[C-11]methyl-L-tryptophan PET maps brain serotonin synthesis and kynurenine pathway metabolism. J Cereb Blood Flow Metab 20:2-9, 2000

6. Chugani HT, Kumar A, Kupsky W, Asano E, Sood S, Juhász C: Clinical and histopathologic correlates of 11C-alpha-methyl-L-tryptophan (AMT) PET abnormalities in children with intractable epilepsy. Epilepsia 52:1692-1698, 2011

7. Dedeurwaerdere S, Friedman A, Fabene PF, Mazarati A, Murashima YL, Vezzani A, et al: Finding a better drug for epilepsy: antiinflammatory targets. Epilepsia 53:1113-1118, 2012

8. Diksic M, Nagahiro S, Sourkes TL: Biological model for the in vivo measurement of rate of serotonin synthesis in the brain. J Neural Transm Suppl 29:131-140, 1990

9. Heyes MP: The kynurenine pathway and neurologic disease. Therapeutic strategies. Adv Exp Med Biol 398:125-129, 1996

10. Hissong BD, Byrne GI, Padilla ML, Carlin JM: Upregulation of interferon-induced indoleamine 2,3-dioxygenase in human macrophage cultures by lipopolysaccharide, muramyl tripeptide, and interleukin-1. Cell Immunol 160:264-269, 1995

11. Ismail FY, Kossoff EH: AERRPS, DESC, NORSE, FIRES: multi-labeling or distinct epileptic entities? Epilepsia 52:e185e189, 2011

12. Iyer A, Zurolo E, Spliet WG, van Rijen PC, Baayen JC, Gorter JA, et al: Evaluation of the innate and adaptive immunity 
in type I and type II focal cortical dysplasias. Epilepsia 51: 1763-1773, 2010

13. Jacobs AH, Tavitian B: Noninvasive molecular imaging of neuroinflammation. J Cereb Blood Flow Metab 32:1393-1415, 2012

14. Juhász C, Chugani DC, Muzik O, Shah A, Asano E, Mangner TJ, et al: Alpha-methyl-L-tryptophan PET detects epileptogenic cortex in children with intractable epilepsy. Neurology 60:960-968, 2003

15. Juhász C, Chugani DC, Muzik O, Wu D, Sloan AE, Barger G, et al: In vivo uptake and metabolism of alpha-[11C]methylL-tryptophan in human brain tumors. J Cereb Blood Flow Metab 26:345-357, 2006

16. Kamson DO, Juhász C, Buth A, Kupsky WJ, Barger GR, Chakraborty PK, et al: Tryptophan PET in pretreatment delineation of newly-diagnosed gliomas: MRI and histopathologic correlates. J Neurooncol 112:121-132, 2013

17. Körtvelyessy P, Lerche H, Weber Y: FIRES and NORSE are distinct entities. Epilepsia 53:1276, 2012

18. Librizzi L, Noè F, Vezzani A, de Curtis M, Ravizza T: Seizureinduced brain-borne inflammation sustains seizure recurrence and blood-brain barrier damage. Ann Neurol 72:82-90, 2012

19. Maldonado M, Baybis M, Newman D, Kolson DL, Chen W, McKhann G II, et al: Expression of ICAM-1, TNF-alpha, NF kappa B, and MAP kinase in tubers of the tuberous sclerosis complex. Neurobiol Dis 14:279-290, 2003

20. Nabbout R, Vezzani A, Dulac O, Chiron C: Acute encephalopathy with inflammation-mediated status epilepticus. Lancet Neurol 10:99-108, 2011

21. O'Connor JC, André C, Wang Y, Lawson MA, Szegedi SS, Lestage J, et al: Interferon-gamma and tumor necrosis factor- alpha mediate the upregulation of indoleamine 2,3-dioxygenase and the induction of depressive-like behavior in mice in response to bacillus Calmette-Guerin. J Neurosci 29:42004209, 2009

22. Ravizza T, Boer K, Redeker S, Spliet WG, van Rijen PC, Troost $\mathrm{D}$, et al: The IL-1beta system in epilepsy-associated malformations of cortical development. Neurobiol Dis 24:128-143, 2006

23. Tohyama Y, Yamane F, Fikre Merid M, Blier P, Diksic M: Effects of serotine receptors agonists, TFMPP and CGS12066B, on regional serotonin synthesis in the rat brain: an autoradiographic study. J Neurochem 80:788-798, 2002

24. Vezzani A, Balosso S, Ravizza T: The role of cytokines in the pathophysiology of epilepsy. Brain Behav Immun 22:797803, 2008

25. Vezzani A, French J, Bartfai T, Baram TZ: The role of inflammation in epilepsy. Nat Rev Neurol 7:31-40, 2011

26. Wilder-Smith EP, Lim EC, Teoh HL, Sharma VK, Tan JJ, Chan BP, et al: The NORSE (new-onset refractory status epilepticus) syndrome: defining a disease entity. Ann Acad Med Singapore 34:417-420, 2005

Manuscript submitted January 23, 2013.

Accepted March 13, 2013.

Please include this information when citing this paper: DOI: 10.3171/2013.3.FOCUS1336.

Address correspondence to: Sandeep Mittal, M.D., F.R.C.S.C., Department of Neurosurgery, Wayne State University, 4160 John R Street, Suite 930, Detroit, Michigan 48201. email: smittal@med. wayne.edu. 